\title{
Accuracy of CT for Measuring Femoral Neck Anteversion in Children With Developmental Dislocation of the Hip Verified Using 3D Printing Technology
}

\section{Zhencun Cai}

General Hospital of Northern Military Area: General Hospital of Northern Theatre command

\section{Chengzhe Piao}

Central Hospital affiliated to Shenyang Medical College

Tianyu Zhang

Central Hospital affiliated to Shenyang Medical College

Lianyong Li

Shengjing Hospital of China Medical University

Liangbi Xiang ( $\square$ xiangliangbi@126.com )

General Hospital of Northern Theatre command

\section{Research Article}

Keywords: femoral neck, anteversion angle, 3D printing technology, CT measurement, hip joint

Posted Date: March 25th, 2021

DOl: https://doi.org/10.21203/rs.3.rs-358038/v1

License: (c) (1) This work is licensed under a Creative Commons Attribution 4.0 International License.

Read Full License

Version of Record: A version of this preprint was published at Journal of Orthopaedic Surgery and Research on April 14th, 2021. See the published version at https://doi.org/10.1186/s13018-021-02400-x. 


\section{Abstract}

Background: Accurate femoral neck anteversion angle (FNA) measurement is of great significance in the diagnosis and treatment of developmental dysplasia of the hip (DDH) in children. The FNA measurement still remains a controversy. We aimed to verify the accuracy of our CT method by 3D printing technology , and to evaluate its clinical value.

Methods: Sixty-eight children with unilateral DDH were enrolled, and their FNA was measured using 2DCT and 3D-CT, respectively, by three observers. This procedure was repeated 3 months later. The above measurement outcomes were then compared with the results in the 3D-printed femur (3D-PF) model. The FNA in the 3D-PF model was measured by three observers collectively through electronic angle instrument.

Results: The primary measurement of FNA at the affected hips by 2D-CT was $44.0 \pm 6.1,49.5 \pm 8.9$ and $52.8 \pm 7.9$ degrees, respectively. On the 3D-CT, it was $47.6 \pm 5.4,49.3 \pm 6.8$ and $48.6 \pm 6.2$ degrees. Three months later, the FNA on 2D-CT was $49.3 \pm 10.5,42.8 \pm 7.4$ and $45.1 \pm 9.3$ degrees, and it was $48.0 \pm 6.5$, $48.9 \pm 7.2$ and $49.0 \pm 5.7$ degrees on 3D-CT, respectively. The FNA in the 3D-PF model at the affected and unaffected hips was $48.5 \pm 6.6$ and $36.9 \pm 13.1$ degrees. There were significant differences between 2D-CT and 3D-PF measurements, but no significant difference was found between 3D-CT and 3D-PF measurements. The results by 2D-CT showed significant differences among groups and between the groups. However, the results by 3D-CT had no significant differences among groups or between the groups.

Conclusion: The results of our study showed that 3D-CT is a more precise, and reproducible method for FNA measurement in DDH. The FNA at the affected hips is 11.6 degree larger than the unaffected in DDH children aged 3-8 years.

\section{Introduction}

Developmental dysplasia of the hip (DDH) is commonly seen in pediatric orthopedics, and femoral neck anteversion angle (FNA) enlargement is one of the important pathological changes of DDH [1, 2]. In 1954, Billing [3]. clearly defined FNA as the angle between the femoral neck axis and condylar plane (determined by the femoral shaft axis and the femoral condyle axis), which has been widely recognized [4]. With technology progresses, it is found that FNA is closely related to the hip stress and dysplasia, and is critical for the choice of surgical methods $[5,6]$. Therefore, how to accurately measure FNA has always been a hotspot in the orthopaedic field.

There are many FNA measuring methods which still remain controversial $[1,7]$. Initially, FNA measurement was usually done by biplane X-ray method. However, biplane X-ray method provides spatial superposition information, and especially, the patient position is uncertain while radiographing, so many scholars believe that this method is inaccurate $[8,9]$. Later, the researchers gradually shifted their focus to how to measure FNA on CT images. In terms of accuracy, reliability and simplicity, the method of 
measuring FNA on CT images is better than biplane X-ray method, but some scholars report that the FNA measured on CT by two-dimensional computed tomography (2D-CT) method may still be different from the true value because the femoral neck is an upward and forward three-dimensional structure [10].

Along with the emergence of three-dimensional reconstruction technology and computer aided design, scholars have designed some three-dimensional computed tomography (3D-CT) measurement methods for FNA based on femoral 3D-CT images [11], and such measurement methods have been widely used in clinic. We also have designed a FNA 3D-CT measurement method [12], and found that this method has good stability, repeatability, and high clinical value. However, the accuracy still raised some doubts [13].

Due to the body particularity of the DDH children, it is difficult for us to obtain real femur specimens for physical measurement. Therefore, the true value of FNA in DDH children and whether the FNA measured by our 3D-CT method is close to the true value have not been verified. In recent years, with the rapid development of 3D printing technology, by CT data, we can print a model exactly the same as the human skeleton [14]. 3D printing technology provides ideas for us to verify the accuracy of CT for FNA measurement in DDH children. In this study, we aimed to measure FNA in DDH children by 3D-CT and 2DCT methods, and compare the measured results with the true value obtained by three-dimensional printed femur (3D-PF), and thus evaluate the accuracy of measuring FNA in DDH children by CT.

\section{Patients And Methods}

\section{Clinical data}

In this study, 68 patients were enrolled, including 20 males and 48 females, aged from 3 to 8 (5.56 \pm 0.81$)$; all the patients had unilateral dislocation of the hip, including 46 patients on the left hip and 22 patients on the right hip. This study was approved by the ethics committee of the Affiliated Central Hospital of Shenyang Medical College, and met the requirements of the declaration of Helsinki. The legal representative of the patient signed the informed consent. The main clinical manifestations of child patients were claudication, pain or hip instability. With X-ray or CT, all the child patients were diagnosed as DDH by two professors. None of the children received any treatment before admission. Cases with neuromuscular diseases and other congenital malformations were excluded.

\section{Measurement method}

The patients were scanned by Philips Brilllance 64-slice spiral CT (scanning conditions: slice thickness: $1.5 \mathrm{~mm}$, slice interval: $0.5 \mathrm{~mm}$, pitch: 0.673 , tube voltage: $120 \mathrm{kv}$, tube current: $70-120 \mathrm{~mA}$ ). The CT scanning ranged from anterior superior iliac spine to femoral condyles, and the CT images were sent to CT workstation (Extended Brilliance Workspace V3.5.0.2250) and the hospital's picture archiving and communication system for measurement. In 2D-CT and 3D-CT measurements, each observer (2 radiologists $\mathrm{A}$ and $\mathrm{B}$, and 1 orthopedist $\mathrm{C}$; all are professors) individually completed the measurements, and measured the CT images of patients for the second time after three months. However, in 3D-PF 
measurement, three observers (A, B and $C$ ) jointly and simultaneously measured the FNA of the normal and dislocated femurs.

\section{D-CT measurement method}

The most commonly used clinical measurement method described in literature reports was selected [15]. A middle-slice CT image showing both the femoral head and neck was selected, on which the femoral head center and the femoral neck center was then connected to determine the femoral head-neck axis; meanwhile, a CT slice image with the two femoral condyles in the largest size was selected, on which the posterior margins of the medial and lateral femoral condyles were then connected. The angle between the femoral head-neck axis and the line connecting the posterior margins of the medial and lateral femoral condyles was the 2D-FNA (Fig. 1).

\section{D-CT measurement method}

After 3D reconstruction of the distal and proximal femoral CT data (Fig. 2A)., the image was rotated to overlap the distal and proximal femurs on one plane, and have the lowest point of greater trochanter in the middle of the lowest points of medial and lateral femoral condyles, and these three lowest points mentioned above on the same horizontal line. The angle between this horizontal line and the line connecting the femoral head center and the midpoint of the narrowest part of the femoral neck is the 3DFNA (Fig. 2B).

\section{D-PF measurement method}

The DICOM data of the whole length of the femur obtained by CT scanning were extracted and imported into the computer, the femur 3D model was reconstructed by Mimics 10.01 software (Materialise, Leuven, Belgium), and then it was reconstructed with masks. We set the ratio of the reconstructed femur size and the actual femur size as 1:1, saved the data of the reconstructed 3D model of the femur in STL format, and then outputted the data to quickly print the physical model of the femur 1:1 through a 3D printer (MakerBot, Brooklyn, New York). The material used in the 3D printed femur model is polylactic acid.

The 1:1 physical model of the femur was placed on the horizontal measurement plane, with the medial and lateral femoral condyles and the posterior margin of the greater trochanter in the same plane. The femoral head center and femoral neck center were determined by vernier caliper. The line connecting the femoral head center and the femoral neck center is the femoral neck axis (observing from proximal to distal). An electronic angle measuring instrument was used to measure the angle between the femoral neck axis and the horizontal plane, and such angle is the 3DPF-FNA (Fig. 3).

\section{Statistical analyses}

SPSS software version 23.0 (SPSS company, Chicago, Illinois) was used for statistical analysis. The consistency of every intra and inter observer between the three groups was analyzed using Pearson correlation coefficient and intra class correlation coefficient (ICC). Based on the bidirectional random effect, absolute consistency and multi rater measurement model, the ICC estimates of 2D-CT and 3D-CT 
measurements and the $95 \%$ confidence intervals $(\mathrm{Cl})$ of inter rater and intra rater reliability were calculated. The interpretation of ICC estimation is as follows: $<0.50$, poor; $0.50-0.75$, average; $0.75-0.90$, good; above 0.90 excellent [16].

Bland Altman diagram was used to evaluate the consistency among 2D-FNA 3D-FNA and 3DPF-FNA. ANOVA with post hoc tests was used to compare the differences among 2D-FNA, 3D-FNA and 3DPF-FNA. Independent student t-test was used to evaluate the difference of FNA angle between dislocated and normal hips. Pearson correlation analysis was used to analyze the correlation between FNAs of dislocated hip and age of children. $P<0.05$ was considered statistically significant.

\section{Results}

\section{Results of FNA in 2D-CT, 3D-CT, and 3D-PF measurement}

For femurs at the dislocated sides of the 68 patients, in the first measurement, the FNA measured by the three observers were $44.0^{\circ} \pm 6.1^{\circ}, 49.5^{\circ} \pm 8.9^{\circ}$ and $52.8^{\circ} \pm 7.9^{\circ}$ on $2 \mathrm{D}-\mathrm{CT}$ respectively, and $47.6^{\circ} \pm 5.4^{\circ}, 49.3^{\circ}$ $\pm 6.8^{\circ}$ and $48.6^{\circ} \pm 6.2^{\circ}$ on $3 \mathrm{D}-\mathrm{CT}$. In the second measurement performed 3 months later, the FNA measured by the three observers were respectively $49.3^{\circ} \pm 10.5^{\circ}, 42.8^{\circ} \pm 7.4^{\circ}$ and $45.1^{\circ} \pm 9.3^{\circ}$ on $2 \mathrm{D}-\mathrm{CT}$, and $48.0^{\circ} \pm 6.5^{\circ}$ $\circ, 48.9^{\circ} \pm 7.2^{\circ}$ and $49.0^{\circ} \pm 5.7^{\circ}$ on $3 \mathrm{D}-\mathrm{CT}$. The FNA measured by $3 \mathrm{D}$-PF was $48.5^{\circ} \pm 6.6^{\circ}$. The measurement results of FNA were significantly different compared with 2D-CT and 3D-CT $(P=.006)$, and the measurement results of 2D-CT and 3D-PF were also significantly different $(P=.007)$. However, there was no significant difference between 3D-CT and 3D-PF $(P=.081)$.

Bland-Altman diagram showed that there was a good consistency in the measurement of FNA in DDH children between the 3D-CT measurement and 3D-PF measurement. However, there was no consistency in 2D-CT measurements, when compared with 3D-CT measurements, or 3D-PF measurements (Fig. 4).

The consistency of intra observer and inter observer was poor in the measurement of FNA by 2D-CT. However, FNA's 3D-CT measurements showed good consistency within the three observers (Table 1). The results showed that the accuracy, stability and repeatability of 2D-CT method were relatively bad, while the reliability and repeatability of 3D-CT method were satisfied.

Table 1 Comparison of intra-observer and inter-observer consistency in the measurement 


\begin{tabular}{|c|c|c|c|c|}
\hline \multirow[t]{2}{*}{ Observer } & \multicolumn{2}{|c|}{ 2D-FNA } & \multicolumn{2}{|l|}{ 3D-FNA } \\
\hline & $\begin{array}{l}\text { ICC } \\
\text { value }\end{array}$ & $95 \% \mathrm{Cl}$ & ICC value & $95 \% \mathrm{Cl}$ \\
\hline$A-A$ & 0.452 & \multirow{2}{*}{$\begin{array}{l}0.386 \text { to } \\
0.498\end{array}$} & 0.959 & 0.931 to 0.986 \\
\hline B-B & 0.512 & & 0.921 & 0.905 to 0.958 \\
\hline C-C & 0.338 & $\begin{array}{l}0.465 \text { to } \\
0.563\end{array}$ & 0.856 & 0.829 to 0.898 \\
\hline A-B & 0.442 & \multirow{2}{*}{$\begin{array}{l}0.296 \text { to } \\
0.385\end{array}$} & 0.890 & 0.875 to 0.921 \\
\hline$B-C$ & 0.496 & & 0.933 & 0.906 to 0.959 \\
\hline \multirow[t]{3}{*}{$A-C$} & 0.393 & $\begin{array}{l}0.375 \text { to } \\
0.499\end{array}$ & 0.968 & 0.943 to 0.991 \\
\hline & & $\begin{array}{l}0.466 \text { to } \\
0.513\end{array}$ & & \\
\hline & & $\begin{array}{l}0.362 \text { to } \\
0.424\end{array}$ & & \\
\hline
\end{tabular}

\section{FNA characteristics of the dislocated hips}

The FNA of the dislocated hip was larger than that of the normal hip by measuring the real measurement of 3D-PF model. For patients aged 3-8 years with DDH, the FNA of normal hip was $36.9^{\circ} \pm 13.1^{\circ}$, and the FNA of dislocated hip was $48.5^{\circ} \pm 6.6^{\circ}$. Statistical analysis showed that the measurement results of both hip joints were significantly different $(P=0.003)$. The FNA of the dislocated hip increased by approximately 11.6 (mean) compared to the normal hip (Table 2).

Table 2 Comparison of FNA in dislocated hip and normal hip groups

\begin{tabular}{lccc}
\hline & $\mathrm{n}$ & $\begin{array}{c}\text { FNA } ;^{\circ} \\
(\text { mean } \pm \text { SD })\end{array}$ & P value \\
\hline normal hips & 68 & $36.9 \pm 13.1$ & \\
dislocated hips & 68 & $48.5 \pm 6.6$ & 0.003 \\
\hline
\end{tabular}

The groups were divided based on age, Pearson correlation analysis showed that there was no correlation between age and FNA in the normal hip group $(r=0.568 ; P=.093)$, while in the dislocated hip group, FNA increased with age, and there was a significant positive correlation between age and FNA $(r=0.672, P$ $=.002)$ (Table 3 ).

Table 3 Relationship between the FNA and the ages of patients 


\begin{tabular}{cccl}
\hline Age group (years) & $\mathrm{n}$ & $\begin{array}{r}\text { normal hips }\left(\mathrm{FNA}^{\circ} \text { ) }\right. \\
(\text { mean } \pm \mathrm{SD})\end{array}$ & $\begin{array}{c}\text { dislocated hips }\left(\mathrm{FNA} ;{ }^{\circ}\right) \\
(\text { mean } \pm \mathrm{SD})\end{array}$ \\
\hline $3-5$ & 22 & $35.9 \pm 9.1$ & $42.7 \pm 9.9$ \\
$5-7$ & 26 & $37.8 \pm 8.3$ & $48.4 \pm 10.7$ \\
$7-8$ & 20 & $36.7 \pm 9.5$ & $51.6 \pm 8.2$ \\
\hline
\end{tabular}

normal hips: $r=0.568 ; P=0.093$. dislocated hips: $r=0.672, P=0.002$.

\section{Discussion}

The treatment for DDH is to achieve concentric reduction of femoral head and acetabulum, so as to restore the normal anatomical structure of hips, which is also the key to evaluate the therapeutic effect after surgeries $[17,18]$. Most scholars believe that the FNA increase is one of the important pathological changes of DDH [19]. Proximal femoral derotation osteotomy is often used in surgeries for children with DDH to correct the large FNA, so as to achieve concentric reduction of femoral head and acetabulum [20, 21]. However, to determine whether or not a rotation is needed or what the rotation angle is, accurate measurement is required before surgeries. The traditional methods mostly depend on the surgeon's experience to determine the osteotomy rotation angle, which is obviously lack of accuracy and will affect the surgery effect [22]. Therefore, finding an accurate and simple method to measure FNA before surgeries is essential.

In the past, many scholars have studied how to accurately measure FNA and invented many FNA measurement methods. However, it is a pity that so far there is no gold standard recognized by most people [23]. At present, CT measurement methods are widely used. There are many different kinds of CT measurement methods [24, 25], where 2D-CT measurement method is the most widely used because of its relatively lower technical requirements, but there is a great controversy about the accuracy of the measurement results. A lot of authors think that 2D-CT method is to use $2 \mathrm{D}$ images to measure the $3 \mathrm{D}$ structure, which is obviously inaccurate, as $2 \mathrm{D}$ images cannot simultaneously show the complete structure of the proximal femur [26]. Besides, in 2D-CT measurement method, it is more random and subjective in selection of CT slice images for measurement, which leads to great differences in measurement results between different physicians $[27,28]$. In this study, it is found that the difference in results between 3D-PF model and 2D-CT measurement was statistically significant, indicating that the accuracy of 2D-CT measurement method was not very well. In the 2D-CT method for the same 68 patients, the results obtained by the three observers were quite different. In addition, in the 2D-CT measurement for the same patient by the same observer, the difference were still statistically significant in results between the first measurement and the second performed 3 months later, indicating that the stability and repeatability of 2D-CT measurement method in FNA measurement were poor. 
We further analyzed the 2D-CT measurement processes by the three observers, and found that for the same patient, the CT slice images selected by the three observers for measurement were not exactly the same. In addition, in the marking for femoral neck axis and line connecting medial and lateral femoral condyles, the mark points selected are also different between observers. Therefore, we believe that the instability, poor accuracy and poor repeatability in FNA measurement results of 2D-CT method may be caused by the difference in selection of CT slice images and mark points between the observers.

With 3D-CT technology, we can reconstruct 2D images into 3D images by imaging software. By rotating the 3D images, we can observe the whole from any angle, and correct the patient position according to the skeletal coordinates before measurement. The 3D-CT FNA measurement method used in this study is our self-designed method based on the characteristics of the femurs [12]. This method is easy to operate and easy to learn. Since applying this method in the clinic, we have achieved good therapeutic effect, because we calculated the rotation angle of proximal femoral osteotomy based on the FNA measured by 3D-CT method before surgeries, and performed osteotomy according to the calculation result mentioned above. Previous studies have shown that the stability and repeatability in measurement results of our 3DCT method are significantly better than that of 2D-CT method, but some scholars still question the accuracy of our measurement results [13]. It is difficult to accurately measure the femur structure in human body, and DDH patients are children, so we cannot take the real femur for accurate FNA measurement. Therefore, we have been unable to get the accurate FNA to test the accuracy of 3D-CT measurement method.

The rapid development of 3D printing technology provides an idea for us to test the accuracy of 3D-CT method in measuring FNA in children with DDH. We collected the CT data of femurs of the 68 children with $\mathrm{DDH}$, and then printed a 1:1 femur model exactly the same as the human skeleton by 3D printing technology, and then performed physical measurement on the model to obtain the accurate FNA of DDH patients. In this study, the FNA in children with DDH measured by 3D-CT method was compared with the real FNA obtained by 3D-PF method, and it was found that there was no statistical difference in the measurement results between 3D-CT method and 3D-PF method, indicating that the FNA in children with DDH measured by 3D-CT method was very accurate.

Whether the FNA on the dislocated sides of DDH patients increases is controversial [29]. By measuring FNA of the 68 patients with 3D-PF method, we found that the average FNA of dislocated hips was $48.5 \pm$ $6.6^{\circ}$, and that of normal hips was $36.9 \pm 13.1^{\circ}$. There was significant difference between the two results as revealed by statistical test, indicating that the FNA on the dislocated hips in children with DDH generally increases, and the FNA on the dislocated hips in 3-8 years old children with DDH is $11.6^{\circ}$ larger than the normal hips. Besides, after assigned by age, we found that compare the 3 years old group with the 8 years old group, the FNA of the normal hips had no significant difference. However, the FNA of the dislocated hips enlarged from $42.7 \pm 9.9^{\circ}$ to $51.6 \pm 8.2^{\circ}$ significantly.

\section{Conclusions}


3D-CT measurement is a precise, beneficial and reproducible method for FNA in DDH. Moreover, this method is easy to operate and easy to learn. The FNA at the affected side is 11.6 degree larger than the healthy side in DDH children aged 3-8 years, and it enlarges with age. In the treatment for children with DDH, we should pay attention to the increase of FNA, and use 3D-CT method to measure FNA to correct FNA timely and accurately.

\section{Declarations}

\section{Funding}

This study was supported by the Foundation of Liaoning Province Education Department (Shenyang Medical College 2019).

\section{Availability of data and materials}

The data and materials used and/or analyzed during the current study are not publicly available but available from the corresponding author on reasonable request.

\section{Ethics approval and consent to participate}

This study was approved by the Ethics Committee of Affiliated Central Hospital of Shenyang Medical College, China.

\section{Conflict of interest}

The authors declare that they have no conflicts of interest.

\section{References}

1. Scorcelletti M, Reeves ND, Rittweger J, et al. Femoral anteversion: significance and measurement [J]. J Anat. 2020;237(5):811-26.

2. Klein C, Fontanarosa A, Khouri N, et al. Anterior and lateral overcoverage after triple pelvic osteotomy in childhood for developmental dislocation of the hip with acetabular dysplasia: Frequency, features, and medium-term clinical impact [J]. Orthop Traumatol Surg Res. 2018;104(3):383-7.

3. Billing L. Roentgen examination of the proximal femur end in children and adolescents; a standardized technique also suitable for determination of the collum-, anteversion-, and epiphyseal angles; a study of slipped epiphysis and coxa plana [J]. Acta Radiol Suppl. 1954;110:1-80.

4. Zhang RY, Su XY, Zhao JX, et a1. Three-dimensional morphological analysis of the femoral neck torsion angle-an anatomical study [J]. J Orthop Surg Res. 2020;15(1):192-6.

5. Kraeutler MJ, Chadayammuri V, Garabekyan T, et al. Femoral Version Abnormalities Significantly outweigh Effect of Cam Impingement on Hip Internal Rotation [J]. J Bone Joint Surg Am. 2018;100(3):205-10. 
6. Boughton OR, Uemura K, Tamura K, et al. Gender and disease severity determine proximal femoral morphology in developmental dysplasia of the hip [J]. J orthop Res. 2019; 37(5):1123-32.

7. Sangeux M, Mahy J, Graham HK. Do physical examination and CT-scan measures of femoral neck anteversion and tibial torsion relate to each other?[J]. Gait Posture. 2014;39(1):12-8.

8. Westberry DE, Wack LI, Davis RB, et al. Femoral anteversion assessment: Comparison of physical examination, gait analysis, and EOS biplanar radiography [J]. Gait Posture. 2018; 62:285-90.

9. Patro BP, Behera S, Das SS, et al. Estimation of Femoral Neck Anteversion in Adults: A Comparison Between Clinical Method, Radiography, and Computed Tomography at a Tertiary-care Center in Eastern India [J]. Cureus. 2019;11(4):e4469-72.

10. Pierrepont JW, Marel E, Baré JV, et a1. Variation in femoral anteversion in patients requiring total hip replacement[J]. Hip Int. 2020;30(3):281-7.

11. Gose S, Sakai T, Shibata T, et al. Morphometric analysis of the femur in cerebral palsy : 3dimensional CT study [J[. J Pediatr Orthop. 2010;30(6):568-74.

12. Jia JJ, Li LL, Zhang LJ, et al. Three dimensional-CT evaluation of femoral neck anteversion, acetabular anteversion and combined anteversion in unilateral DDH in an early walking age group [J]. Int Orthop. 2012;36(1):119-24.

13. Mootha AK, Saini R. Comment on Jia et al. Three dimensional CT evaluation of femoral neck anteversion, acetabular anteversion and combined anteversion in unilateral DDH in an early walking age group [J]. Int Orthop. 2012;36(7):1531-2.

14. Cho W, Job AV, Chen J, et al. A Review of Current Clinical Applications of Three-Dimensional Printing in Spine Surgery [J]. Asian Spine J. 2018;12(1):171-7.

15. Weiner DS, Cook AJ, Hoyt WA, et al. Computed tomography in the measurement of femoral anteversion [J]. Orthopedics. 1978;1(4):299-306.

16. Koo TK, Li MY. A guideline of selecting and reporting intraclass correlation coefficients for reliability research [J]. J Chiropr Med. 2016;15(2):155-63.

17. Wyles CC, Vargas JS, Heidenreich MJ, et al. Natural history of the dysplastic hip following modern periacetabular osteotomy [J]. J Bone Joint Surg Am. 2019;101(10):932-8.

18. Thawrani D, Sucato DJ, Podeszwa DA, et al. Complications associated with the Berneseperiacetabular osteotomy for hip dysplasia in adolescents [J]. J Bone Joint Surg Am. 2010;92(8):1707-14.

19. Tarpada SP, Girdler SJ, Morris MT. Developmental dysplasia of the hip: a history of innovation [J]. J Pediatr Orthop B. 2018;27(3):271-3.

20. Kothari A, Grammatopoulos G, Hopewell S, et al. How does bony surgery affect results of anterior open reduction in walking-age children with developmental hip dysplasia [J]. Clin Orthop Relat Res. 2016;474(5):1199-208.

21. Qadir I, Ahmad S, Zaman AU, et al. One-stage Hip Reconstruction for Developmental Hip Dysplasia in Children over 8 Years of Age [J]. Hip Pelvis. 2018;30(4):260-8. 
22. Bhuyan BK. Outcome of one-stage treatment of developmental dysplasia of hip in older children [J]. Indian J Orthop. 2012;46(5):548-55.

23. Kaiser $P$, Attal R, Kammerer M, et al. Significant differences in femoral torsion values depending on the CT measurement technique [J]. Arch Orthop Trauma Sung. 2016;136(9): 1259-64.

24. Chimhundu C, Sivarasu S, Steiner S, et al. Femoral neck anteversion measurement using linear slot scanning radiography [J]. Med EngPhys. 2016;38(2):187-91》

25. Wells J, Nepple JJ, Crook K. Femoral Morphology in the Dysplastic Hip囚Three-dimensional characterizations with CT [J]. Clin Orthop Relat Res. 2017;475(4):1045-54.

26. Kim JS, Park TS, Park SB, et al. Measurement of femoral neck anteversion in 3D. Part 1: 3D imaging method [J]. Med Biol Eng Comput. 2000;38(6):603-9.

27. Park KK, Tsai TY, Dimitriou D, et a1. Utility of Preoperative Femoral Neck Geometry in Predicting Femoral Stem Anteversion [J]. J Arthroplasty. 2015;30(6):1079-84.

28. Sung $\mathrm{KH}$, Youn $\mathrm{K}$, Chung $\mathrm{CY}$, et al. Development and Validation of a Mobile Application for Measuring Femoral Anteversion in Patients With Cerebral Palsy [J]. J Pediatr Orthop. 2020;40(6):e516-21.

29. Uota S, Morikita I, Shimokochi Y. Validity and clinical significance of a clinical method to measure femoral anteversion [J]. J Sports Med Phys Fitness. 2019;59(11):1908-14.

\section{Figures}




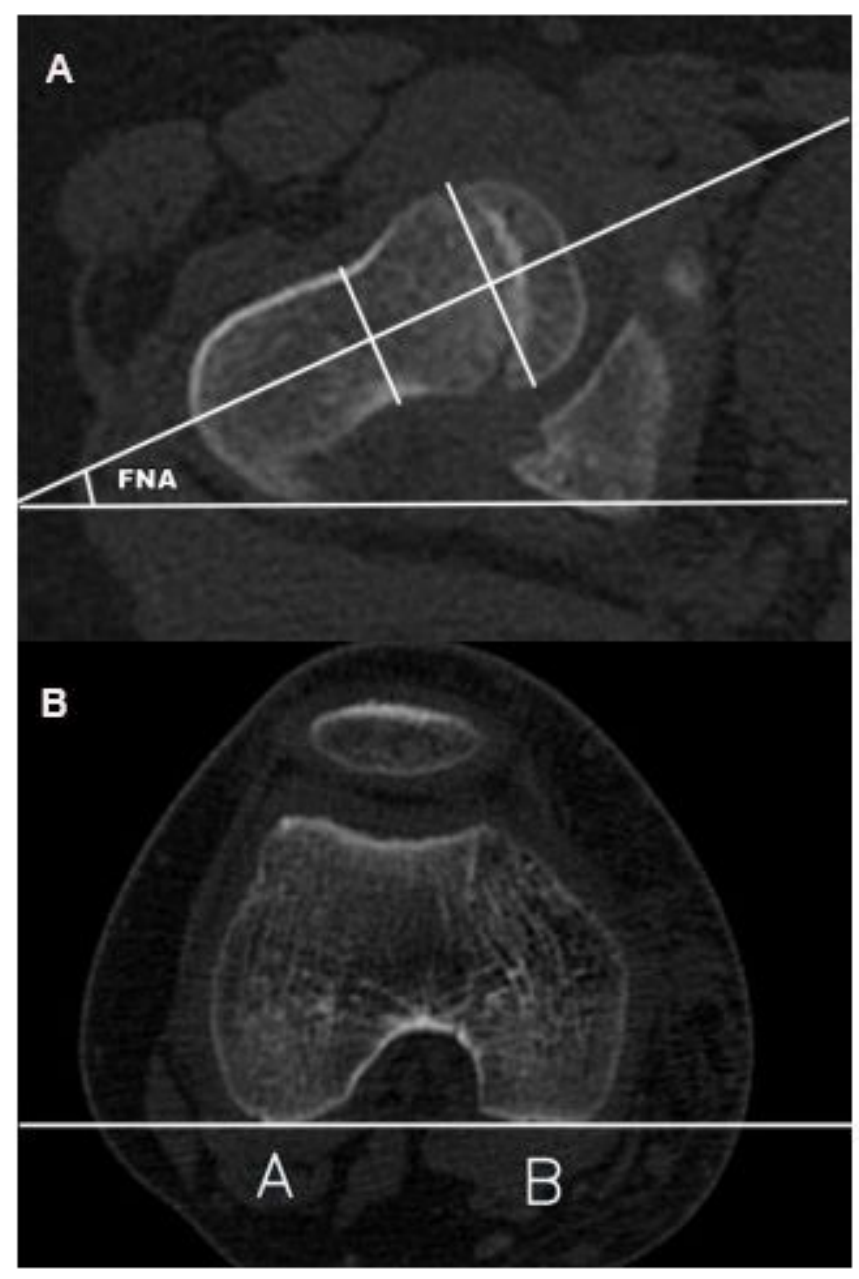

\section{Figure 1}

2D-FNA. (A) CT image of the femoral head and the femoral neck. The center of the femoral head and the femoral neck is connected to determine the the femoral head-neck axis. (B) It shows the largest layer of the two femoral condyles, the points of $A$ and $B$ are the tangent of the posterior rim of the two condyles. The angle between the femoral head-neck axis and the tangent is 2D-FNA. 


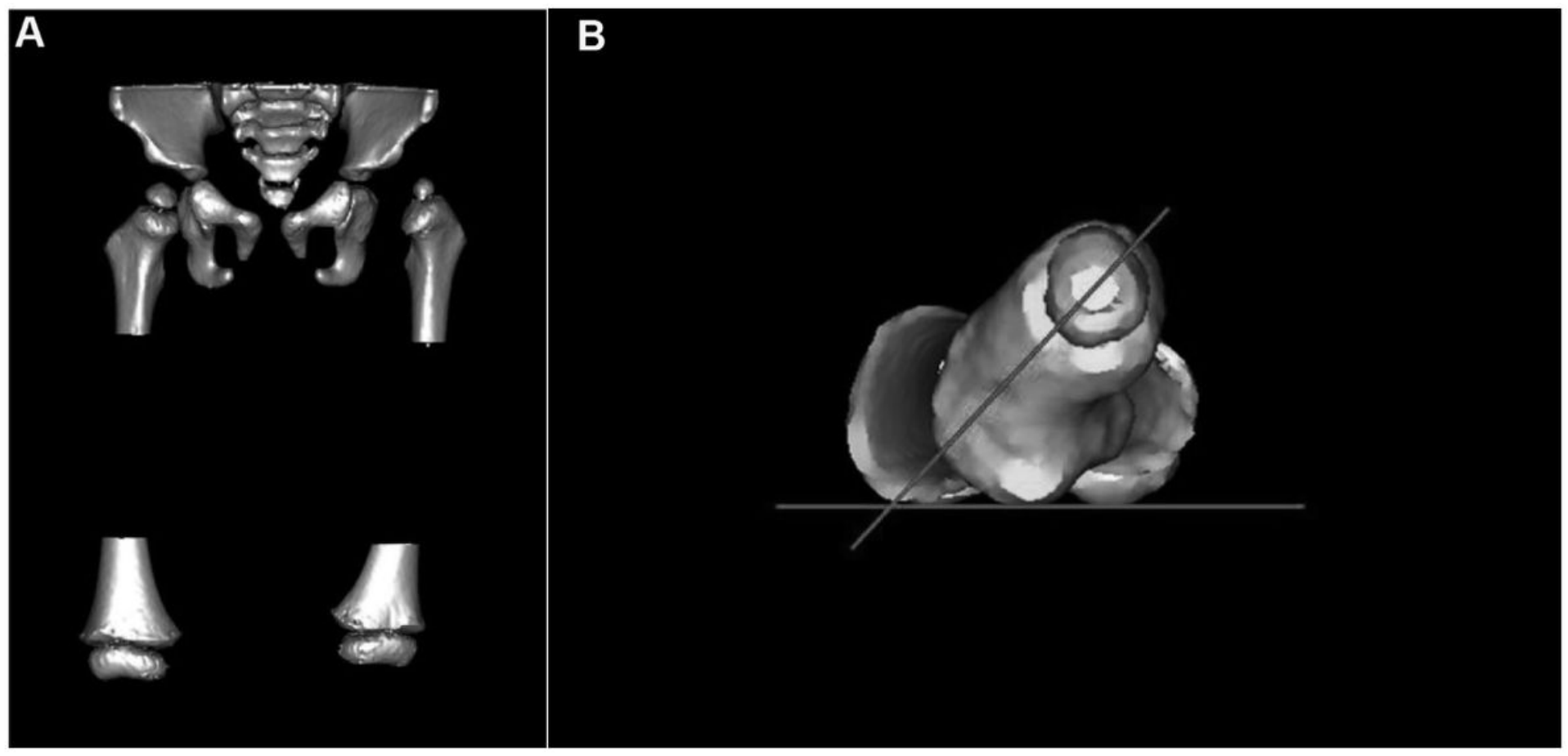

\section{Figure 2}

3D-FNA. (A) CT-reconstruction image of the pelvis and the distal and proximal femur. (B) 3D-FNA: The lowest point of the greater trochanter and the medial and lateral femoral condyles are located on a horizontal line by rotating the image. The angle between this horizontal line and the femoral neck axis is the 3D-FNA. 


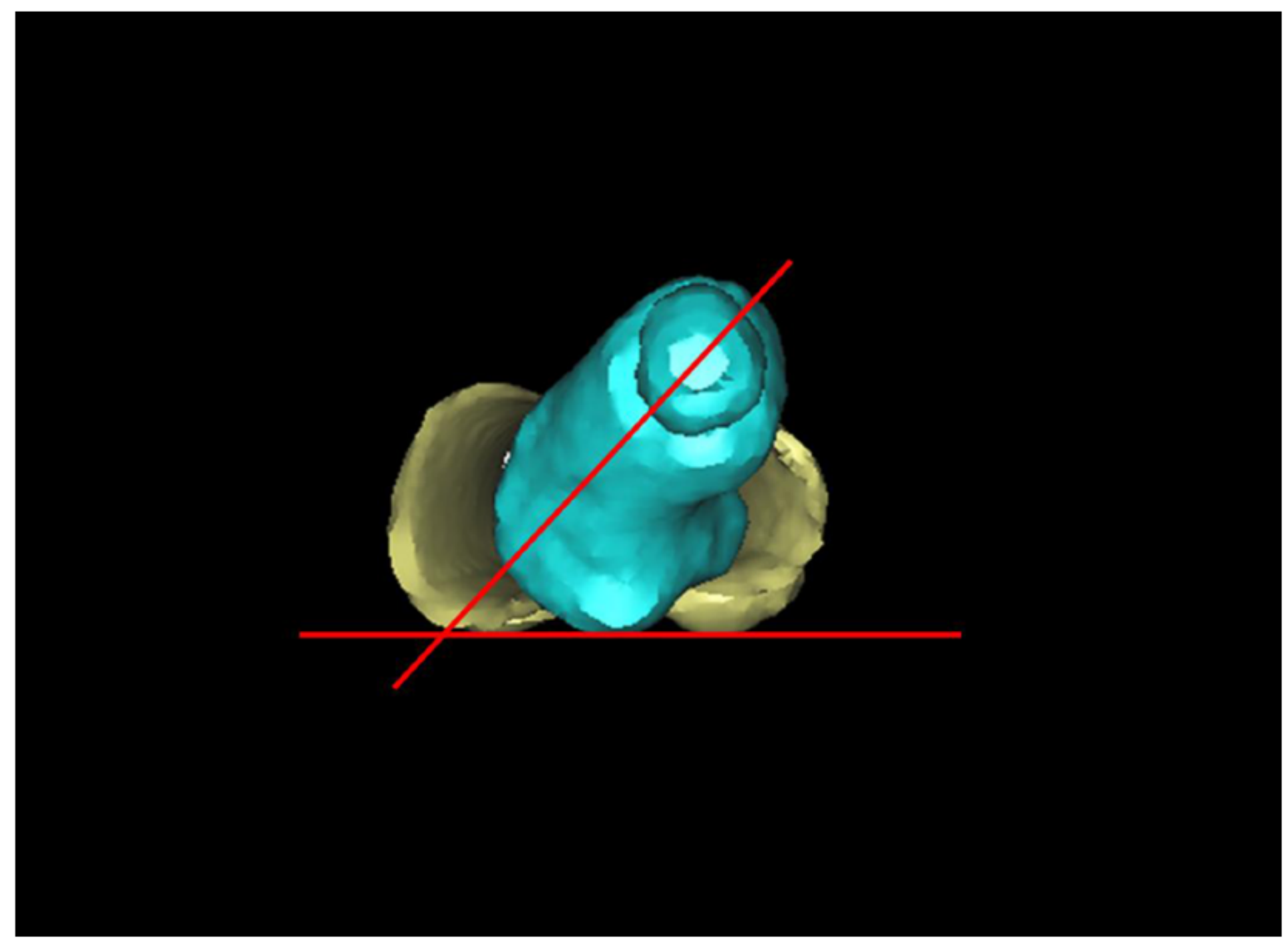

Figure 3

3D PF-FNA. The lowest point of the greater trochanter on the 3D-PF model is located on the same horizontal plane as the medial and lateral condyles of the femur. The center of the femoral head and neck is positioned with a vernier caliper. The angle between these two points and the horizontal plane is measured by electronic angle instrument (called 3DPF-FNA). 

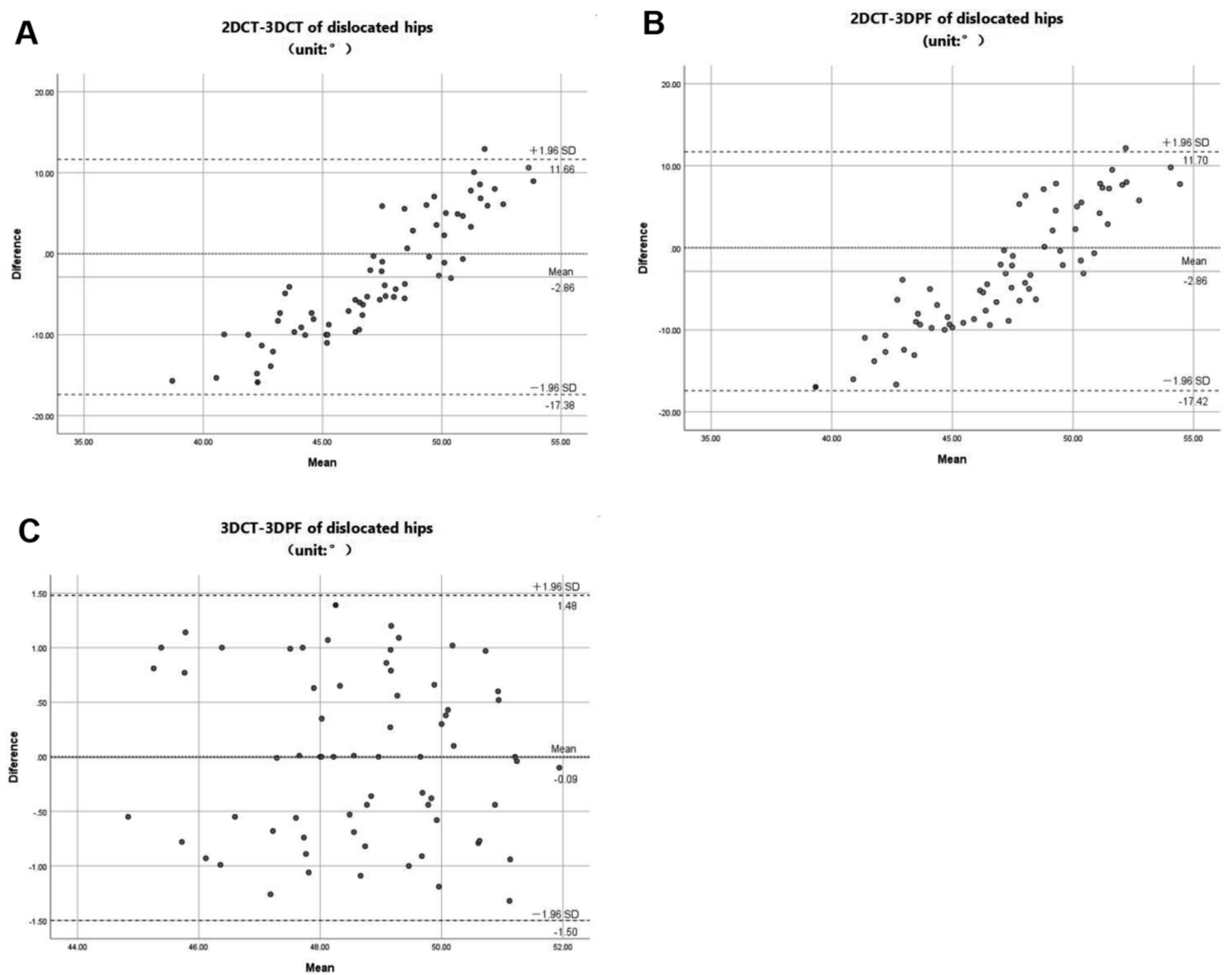

\section{Figure 4}

Bland-Altman analysis of different measurements. (A) Compared with the value measured by 2D-CT and $3 \mathrm{D}-\mathrm{CT}$, the maximum difference is 15.87 and the mean is 47.06 . The results of the two methods are inconsistent. (B) Compared with the value measured by 2D-CT and 3D-PF, the maximum difference is 16.96 and the mean is 47.28 . The results of the two methods are inconsistent. Compared with the value measured by 3D-CT and 3D-PF, the maximum difference is 1.39 , and the mean is 48.71 . The results of the two methods have high consistency. 